\title{
The reduction of birth weight by fine particulate matter and its modification by maternal and neighbourhood-level factors: a multilevel analysis in British Columbia, Canada
}

\author{
Anders C. Erickson ${ }^{1}$, Aleck Ostry ${ }^{2}$, Laurie H. M. Chan ${ }^{3}$ and Laura Arbour ${ }^{1,4^{*}}$
}

\begin{abstract}
Background: The purpose of this research was to determine the relationship between modeled particulate matter $\left(\mathrm{PM}_{2.5}\right)$ exposure and birth weight, including the potential modification by maternal risk factors and indicators of socioeconomic status (SES).

Methods: Birth records from 2001 to 2006 ( $N=231,929)$ were linked to modeled PM 2.5 data from a national land-use regression model along with neighbourhood-level SES and socio-demographic data using 6-digit residential postal codes. Multilevel random coefficient models were used to estimate the effects of $\mathrm{PM}_{2.5}$, SES and other individual and neighbourhood-level covariates on continuous birth weight and test interactions. Gestational age was modeled with a random slope to assess potential neighbourhood-level differences of its effect on birth weight and whether any between-neighbourhood variability can be explained by cross-level interactions.

Results: Models adjusted for individual and neighbourhood-level covariates showed a significant non-linear negative association between $\mathrm{PM}_{2.5}$ and birth weight explaining $8.5 \%$ of the between-neighbourhood differences in mean birth weight. A significant interaction between SES and $\mathrm{PM}_{2.5}$ was observed, revealing a more pronounced negative effect of $\mathrm{PM}_{2.5}$ on birth weight in lower SES neighbourhoods. Further positive and negative modification of the $\mathrm{PM}_{2.5}$ effect was observed with maternal smoking, maternal age, gestational diabetes, and suspected maternal drug or alcohol use. The random intercept variance indicating between-neighbourhood birth weight differences was reduced by $75 \%$ in the final model, while the random slope variance for between-neighbourhood gestational age effects remained virtually unchanged.

Conclusion: We provide evidence that neighbourhood-level SES variables and $\mathrm{PM}_{2.5}$ have both independent and interacting associations with birth weight, and together account for $49 \%$ of the between-neighbourhood differences in birth weight. Evidence of effect modification of $\mathrm{PM}_{2.5}$ on birth weight across various maternal and neighbourhood-level factors suggests that certain sub-populations may be more or less vulnerable to relatively low doses $\mathrm{PM}_{2.5}$ exposure.
\end{abstract}

Keywords: Birth weight, Air pollution, Multilevel model, Neighbourhood effects, Particulate matter, Socioeconomic status, Effect modification

\footnotetext{
* Correspondence: larbour@uvic.ca

${ }^{1}$ Division of Medical Sciences, University of Victoria, Medical Science Bld.

Rm-104, PO Box 1700 STN CSC, Victoria V8W 2Y2 BC, Canada

${ }^{4}$ Department of Medical Genetics, University of British Columbia, C201 - 4500

Oak Street, Vancouver V6H 3N1 BC, Canada

Full list of author information is available at the end of the article
} 


\section{Background}

Studies of exposure to particulate air pollution have consistently shown an association with low birth weight, a predictor of fetal growth restriction and important determinant of infant and child-wellbeing [1-3]. The fine fraction of particulate matter $\left(\mathrm{PM}_{2.5}\right.$ - less than $2.5 \mu \mathrm{m})$ is a complex mixture of elemental and organic carbon compounds, metals and gases that stem predominantly from vehicle exhaust, residential heating and industrial emissions. $\mathrm{PM}_{2.5}$, which includes ultrafine particles less than $0.1 \mu \mathrm{m}$, can penetrate deep into the pulmonary alveolar tissue where inflammatory mediators and possibly the particles themselves translocate into the bloodstream causing systemic cardiovascular and immunological alterations such as platelet activation, coagulation and endothelial dysfunction [4-6]. These physiological changes extend to the placenta, a highly vascularized organ and extension of the maternal cardiovascular system with similarly affected endothelial cellular tissues particularly susceptible to oxidative and inflammatory injury [7-9]. Excess or uncontrolled oxidative stress and inflammation early in pregnancy may disrupt placental cell growth and differentiation, potentially leading to deficient deep placentation and morphological adaptations associated with several adverse pregnancy outcomes including fetal growth restriction [10].

These mechanisms by which $\mathrm{PM}_{2.5}$ may act to adversely impact the reproductive system are not fully understood; however, evidence supports the potential for a shared mode of developmental toxicity with several other known risk factors $[5,11]$. This includes factors that also promote or are associated with oxidative stress and inflammation such as smoking [12], drug use [13], advanced maternal age [14] gestational diabetes [15], and low socioeconomic status (SES) in general [16]. The causal pathways in which SES contributes to adverse pregnancy outcomes can be conceptualized in terms of 'downstream' or mediating exposures, stresses and behaviours acting on the individual through 'upstream' society-level determinants such as poverty, poor education, income inequality and social discrimination/marginalization over the lifespan $[17,18]$. This impact of the social environment on health behaviours and outcomes creates hierarchical structures within which individuals are nested in neighbourhoods and communities with their own set of attributes that can promote or antagonize health and healthy behaviours [19, 20].

A particular challenge in environmental epidemiology is handling data at differing geographic scales. Birth registries and vital statistics provide data on individual births and certain risk factors, but may not have data on socially patterned risk factors. Alternatively, reliable SES data such as education, income and housing quality are often only available from national census databases using arbitrary administrative spatial units. Finally, obtaining individual-level environmental exposure data is often not possible. Therefore, the epidemiologist is often left with a mix of individual-level observations clustered within neighbourhood areas each with distinct attributes. The use of multilevel statistical models separates the individual-level effects from the context of their social and physical environments and can therefore quantify the degree of clustering of individuals within neighbourhood areas and test whether neighbourhood factors themselves have direct effects on the health outcome or act indirectly via the modification of individual-level variables [21, 22].

Through the mechanisms of oxidative stress and inflammation there is evidence that SES may not only confound but modify the $\mathrm{PM}_{2.5}$-birth outcome relationship [23-25]. Various exposures and experiences may act in a non-additive manner to influence fetal development [5]. We present a multilevel cross-sectional analysis of the association between birth weight and $\mathrm{PM}_{2.5}$ in British Columbia, Canada where levels of $\mathrm{PM}_{2.5}$ are relatively low but can vary substantially between different communities [26]. We explore the potential for between-neighbourhood variability for the slope of gestational age on birth weight and whether interactions with $\mathrm{PM}_{2.5}$, neighbourhood-level SES indicators, and/or individual-level risk factors are able to explain any neighbourhood-level variability. We had three research questions: 1) does exposure to $\mathrm{PM}_{2.5}$ and residence in low SES neighbourhoods in $\mathrm{BC}$ have significant independent negative associations with birth weight? 2) does the effect of gestational age on birth weight differ between neighbourhoods? 3) does $\mathrm{PM}_{2.5}$ interact with neighbourhood-level SES and/or individual-level risk factors to modify their independent effects on birth weight to help explain any neighbourhood-level differences?

\section{Methods}

This was a population-based retrospective cohort of singleton births in British Columbia from 2001 to 2006 $(N=237,470)$. Data from the BC Perinatal Data Registry were provided by Perinatal Services British Columbia (PSBC) which included information on maternal-infant health status and outcomes, reproductive history, maternal risk factors and attributes, and residential postal codes. The Registry accounts for $99 \%$ of births and stillbirths in $\mathrm{BC}$ of at least 20 weeks gestation or at least $500 \mathrm{~g}$ birth weight. Research data access is provided by a Partnership Accord /Memorandum of Agreement between all BC Health Authorities and PSBC through the Freedom of Information and Privacy Protection Act [27]. Research ethics board approval was granted by the University of Victoria (ethics protocol \#: 11-043).

The outcome variable was continuous birth weight of singleton births. In order to avoid potential selection bias, we included all births (stillbirth and live) for all 
gestational ages (20-44 weeks). Excluded records included out-of-province and invalid postal codes $(n=1096)$, nonviable births prior to 20 weeks gestation or $<500 \mathrm{~g}(n=14)$, and the list-wise deletion of births missing important data including: cigarettes smoked per day (cigs/day, $n=2501$ ), $\mathrm{PM}_{2.5}(n=1510)$, gestational age $(n=373)$, and birth weight $(n=41)$. All continuous variables, except cigarettes/day, were standardized and centred to ease interpretation and aid model convergence.

The spatial location of each birth record was geocoded based on the latitude-longitude coordinate of the mother's residential postal code at the time of delivery using GeoRef [28]. Birth records were related to their corresponding census dissemination area (DA) by performing a point-in-polygon spatial join procedure in ArcGIS 10.2 [29]. DAs are the smallest geographical unit for which census data are available and represent neighbourhood blocks ranging between 200 and 800 people. While DAs do not necessarily represent existing neighbourhood communities [30], they can act as proxies for a general catchment area of personal home-life activities $[31,32]$. Birth records were identified as being either rural or urban using the Statistics Canada Metropolitan Influence Zone (MIZ) codes which are based on commuting flows of small towns into larger cities and metropolitan areas [33].

Exposure to $\mathrm{PM}_{2.5}$ was estimated using a national land-use regression (LUR) model developed to estimate $\mathrm{PM}_{2.5}$ at the census street block-face level [34]. The model used a number of predictors including satellite measures, proximity to major roads and industry to account for $46 \%$ of the variability in measured annual $\mathrm{PM}_{2.5}$ concentrations. Unlike nitrogen dioxide $\left(\mathrm{NO}_{2}\right), \mathrm{PM}_{2.5}$ tends to have a more homogeneous intra-urban distribution between personal, indoor and ambient exposure [35]. The LUR model estimates used for this study showed very little variability of $\mathrm{PM}_{2.5}$ exposures between individuals within a given DA. We therefore aggregated the point-level estimates of $\mathrm{PM}_{2.5}$ to their DA-level mean and related it to individual birth records as an area-level variable.

The DA-level SES and demographic data were represented by three related but independent datasets all based on the 2006 Statistics Canada national census. The first was a Canadian SES index (SESi) developed by Chan et al. [36]. The second was an education variable representing the proportion of population over 15 with any post-secondary education, including college, trades, or university. The third was the proportion of continental Asian immigrants by DA as it has been shown in BC and elsewhere that healthy babies from Asian and South Asian backgrounds are constitutionally smaller compared to their Caucasian counterparts $[37,38]$. Asian and South Asian ethnicities are well-represented throughout BC but particularly in concentrated pockets throughout the major urban center of Metro Vancouver where levels of $\mathrm{PM}_{2.5}$ are also high. The correlation between immigrant density with SESi and $\mathrm{PM}_{2.5}$ was -0.62 and 0.53 respectively $(p<0.001)$; we therefore created a residual immigrant density variable using a sequential regression technique [39]. Here, immigrant density was regressed against SESi and $\mathrm{PM}_{2.5}$ with the saved residuals representing the uncorrelated and independent contribution of immigrant density on birth weight freed from its collinearity with SESi and $\mathrm{PM}_{2.5}$. This same method was used between SESi and education $(r=0.25)$ creating a residual education variable. The education and immigrant data were obtained by access to ABACUS via the Data Liberation Initiative [40].

In order to avoid data loss from rural DAs, imputation for missing SES, education and immigrant density values was performed. Taking advantage of the nested hierarchical structure of the administrative census and health boundaries, the mean value for a larger encompassing census subdivision (CSD) or local health area (LHA) was imputed for a nested DA with a missing value. There were 1441 values imputed in 52 DAs for SESi $(0.6 \%$ of final N, $0.8 \%$ of DAs), and 3170 values imputed in 108 DAs for both education and immigrant density (1.4 \% of final N, $1.7 \%$ of DAs). Sensitivity analyses were performed using only the non-missing data.

Hierarchical (multilevel) linear regression models were used to test our research questions, thereby accounting for the clustering, or non-independence, of individuals (level-1) belonging to a given DA neighbourhood (level2). The multilevel model allows the intercept and slope to act as random parameters having between-area (DA) variability from an overall (BC-wide) mean intercept and slope. That is, each DA has its own intercept and slope in which their variability from the overall intercept and slope can be investigated with the addition of level-1 and level-2 variables and their interactions [41]. We followed a bottom-up approach to model building to quantify the explained proportional change in variance (PCV), the multilevel model equivalent to an $R^{2}$ [22]. We started with an empty (null) random intercept model without any independent variables in which birth weight is only a function of the mother's residential DA. The presence of significant random intercept variance indicates there are unexplained between-neighbourhood differences in mean birth weight. The proportion of the total variance in birth weight that arises due to neighbourhood differences can be quantified by computing the intra-class correlation (ICC), and hence provides the degree of clustering of individual birth weight within neighbourhoods [22].

Gestational age was added to the null model and given a random slope (i.e., the mean within-DA effect of gestational age on birth weight was allowed to differ between DAs). 
The presence of a significant random slope indicates that its effect is not constant (or equal) for all DAs. Subsequent models included the individual and DA-level variables along with cross-level and within-level interactions in order to assess their fixed effects on birth weight but to also determine if their inclusion addressed any unexplained intercept or slope variance. Models were tested using the Akaike Information Criterion (AIC) to evaluate model performance. All statistical analyses were conducted in Stata 13IC [42].

Finally, while multilevel models address intra-area dependence while quantifying inter-area variance, they assume spatial independence among neighbouring areas. However environmental and social processes can extend beyond arbitrary neighbourhood boundaries. Additionally as mentioned above, census DAs do not necessarily represent neighbourhood dynamics, services, infrastructure, etc.; and evidence of spatial clustering between DAs may indicate that an alternative neighbourhood areal unit should be considered. We used spatial methods to test for this by checking the level-1 model residuals and level-2 predicted random parameters (intercepts and slopes) for spatial autocorrelation using the local Moran's I statistic [43]. The presence of significant residual spatial autocorrelation indicates the existence of unobserved spatial processes causing DAs to cluster and can be a sign of model misspecification. Prediction of the DA-level random intercept and slope errors used an Empirical Bayes method [44] available as a post-estimation command in Stata 13IC.

\section{Results}

After exclusions there were 231,929 singleton (live and stillborn) births located in 6338 neighbourhood DAs (min. = 1, max. $=781$, avg. $=37$ ). Table 1 summarizes the untransformed individual and neighbourhood covariates (non-centered, non-standardized) along with their relationship to $\mathrm{PM}_{2.5}$. Table 2 reports the adjusted coefficients for the individual and DA-level covariate fixed effects on continuous birth weight (Model 1 to 3). Gestational age was modeled using a quadratic term to account for the rapid fetal growth in mid-gestations and its slower growth post-term (>36 weeks). Maternal smoking (cigarettes/day) and $\mathrm{PM}_{2.5}$

Table 1 Descriptive statistics ${ }^{a}$ for individual (Level-1) and DA (Level-2) covariates

\begin{tabular}{|c|c|c|c|c|c|}
\hline \multirow[t]{2}{*}{ Variables } & \multirow[t]{2}{*}{ Mean } & \multirow[t]{2}{*}{ Std. Dev. } & \multirow[t]{2}{*}{ Min-Max } & \multicolumn{2}{|l|}{ PM 2.5 mean $(\mathrm{SE})^{\mathrm{b}}$} \\
\hline & & & & Absence/1st quintile & Presence/5th quintile \\
\hline \multicolumn{6}{|l|}{ Level-1 (individual) } \\
\hline Birth weight (grams) & 3433.3 & 566.51 & $135-6475$ & $7.30(.016)$ & $7.36(.018)^{\mathrm{c}, \mathrm{e}}$ \\
\hline Gestational age (weeks) & 38.8 & 2.02 & $19-44$ & $7.30(.016)$ & $7.30(.017)^{f}$ \\
\hline Maternal age (years) & 29.8 & 5.60 & $11-55$ & $7.10(.022)$ & $7.39(.014)^{c}$ \\
\hline Nulliparous & 0.45 & 0.50 & $0-1$ & $7.27(.016)$ & $7.34(.016)^{c}$ \\
\hline Gestational diabetes & 0.06 & 0.25 & $0-1$ & $7.29(.016)$ & $7.53(.016)^{c}$ \\
\hline Pre-existing diabetes & 0.004 & 0.06 & $0-1$ & $7.30(.016)$ & $7.34(.034)$ \\
\hline Gestational hypertension & 0.02 & 0.15 & $0-1$ & $7.30(.016)$ & $7.44(.019)^{c}$ \\
\hline Poor prenatal care & 0.09 & 0.29 & $0-1$ & $7.28(.016)$ & $7.47(.02)^{c}$ \\
\hline Drug/Alcohol flag & 0.02 & 0.15 & $0-1$ & $7.31(.016)$ & $7.08(.026)^{c}$ \\
\hline Cigarettes/day & 0.79 & 2.91 & $0-20$ & $7.33(.015)$ & $7.02(.023)^{c, g}$ \\
\hline Fall/Winter season & 0.48 & 0.50 & $0-1$ & $7.29(.016)$ & $7.31(.016)^{c}$ \\
\hline \multicolumn{6}{|l|}{ Level-2 (DA) variables } \\
\hline SESi & -0.08 & 0.58 & $-2.22-1.18$ & $7.82(.027)$ & $6.95(.032)^{c, d}$ \\
\hline Higher education & 0.50 & 0.12 & $0-0.95$ & $7.16(.04)$ & $7.53(.022)^{c, d}$ \\
\hline Immigrant density & 0.16 & 0.19 & $0-0.86$ & $6.75(.023)$ & $7.95(.021)^{c, d}$ \\
\hline Rural address & 0.11 & 0.32 & $0-1$ & $7.39(.014)$ & $6.59(.061)^{c, d}$ \\
\hline $\mathrm{PM}_{2.5}\left(\mu \mathrm{g} / \mathrm{m}^{3}\right)$ & 7.30 & 0.86 & $4.41-10.23$ & - & - \\
\hline
\end{tabular}

${ }^{a}$ Values shown are unstandardized, non-centered; Birth weights below 500 grams were included if their gestational age was $>19$ weeks. Gestational age under 20 weeks were included if their birth weight was $>499$ grams. Poor prenatal care: having less than 4 prenatal care visits or was missing; Drug/Alcohol flag: indicates whether physician lists patient's use of alcohol or drugs (prescription, nonprescription, illicit) as a risk factor in this pregnancy; Cigarettes/day: self-reported number of cigarettes smoked daily at 1st prenatal visit (excluding non-smokers, mean $(\mathrm{sd})=7.7(5.41)$ ); Fall/Winter season: birth month $=$ September to February

${ }^{\mathrm{b}}$ Robust standard errors adjusted for 6338 DA clusters

'Significant difference at $p<0.05$ using Wald tests

${ }^{\mathrm{d}} 1$ st vs. 5th quintile

eNormal birth weight vs. low birth weight

fTerm birth vs. preterm birth

${ }^{9}$ Non-smoker vs. current smoker 
Table 2 Adjusted individual and DA-level fixed effects on continuous birth weight

\begin{tabular}{|c|c|c|c|}
\hline \multirow[t]{2}{*}{ Variables } & Model-1 & Model-2 & Model-3 \\
\hline & $\beta(95 \% \mathrm{Cl})$ & $\beta(95 \% \mathrm{Cl})$ & $\beta(95 \% \mathrm{Cl})$ \\
\hline \multicolumn{4}{|l|}{ Level-1 (individual) } \\
\hline Gestational age & $310.2(307.6-312.7)$ & $308.7(306.1-311.2)$ & $308.5(306.0-311.1)$ \\
\hline Gestational age $e^{a}$ & $-11.6(-12.2--11.1)$ & $-11.9(-12.4--11.3)$ & $-11.9(-12.5--11.4)$ \\
\hline Maternal age & $-6.6(-8.6--4.7)$ & $-6.0(-8.0--4.0)$ & $-4.7(-6.6--2.7)$ \\
\hline Nulliparous & $-137.2(-141.0--133.4)$ & $-135.7(-139.5--131.9)$ & $-134.8(-138.6--131.1)$ \\
\hline Gestational diabetes & $54.5(47.1-61.8)$ & $60.0(52.7-67.4)$ & $62.0(54.6-69.4)$ \\
\hline Pre-existing diabetes & $320.7(292.1-349.3)$ & $320.6(292.1-349.1)$ & $321.2(292.6-349.7)$ \\
\hline Gestational hypertension & $-90.1(-102.3--77.9)$ & $-88.9(-101.0--76.7)$ & $-87.6(-99.7--75.4)$ \\
\hline Prenatal care visits & $-59.0(-65.3--52.8)$ & $-55.0(-61.2--48.7)$ & $-52.2(-58.4--45.9)$ \\
\hline Drug/Alcohol flag & $-79.1(-91.2--67.1)$ & $-79.2(-91.2--67.2)$ & $-81.7(-93.7--69.7)$ \\
\hline Cigarettes/day & $-20.8(-22.5--19.0)$ & $-22.0(-23.8--20.3)$ & $-22.7(-24.4--20.9)$ \\
\hline Cigarettes/day ${ }^{a}$ & $0.63(0.51-0.74)$ & $0.68(0.57-0.79)$ & $0.7(0.59-0.82)$ \\
\hline Fall/Winter season & -- & -- & $-6.8(-10.4--3.2)$ \\
\hline \multicolumn{4}{|l|}{ Level-2 (DA) } \\
\hline SESi & -- & $37.4(35.2-39.7)$ & $29.4(27.0-31.8)$ \\
\hline Higher education & -- & $-2.1(-4.4-0.2)$ & $3.0(0.7-5.3)$ \\
\hline Immigrant density & -- & $-29.2(-31.4--26.9)$ & $-31.3(-33.5--29.1)$ \\
\hline Rural address & -- & $4.8(-3.4-12.9)$ & $-14.6(-22.6--6.7)$ \\
\hline $\mathrm{PM}_{2.5}$ & -- & -- & $-23.9(-26.5--21.3)$ \\
\hline $\mathrm{PM}_{2.5}{ }^{\mathrm{a}}$ & -- & -- & $2.8(1.3-4.3)$ \\
\hline
\end{tabular}

See Table 1 legend for variable definitions; ${ }^{2}$ Variables were modeled as quadratics

were also modeled using a quadratic terms, both indicating a subdued dose-response with increasing exposure.

Model 2 added the DA-level variables of SESi, education, immigrant density and rural residence. Their fixed effects show that lower SES and higher Asian immigrant density were significantly associated with lower birth weights (Table 2). Rural DAs and DAs with higher proportion of post-secondary education were not significantly associated with birth weight in this model. However, both became significant after the addition of $\mathrm{PM}_{2.5}$ and season of birth (cold vs. warm) in Model 3. Higher education had a positive association with birth weight, while rural areas had a significant negative association with birth weight. $\mathrm{PM}_{2.5}$ was found to have a significant non-linear negative association on birth weight whereby the negative effect tapers off at higher concentrations of $\mathrm{PM}_{2.5}$ (Fig. 1). Being born in a cold (fall or winter) month also had a significant negative association with birth weight (Table 2).

Model 4 tested interactions with $\mathrm{PM}_{2.5}$ including crosslevel (level-1 by level2) and level-2 by level-2 interactions to explain the between-DA random intercept variability. The model results are presented in Tables 3 and 4 including the main effects as well as the interaction effects with $\mathrm{PM}_{2.5}$. Four maternal variables showed effect modification with $\mathrm{PM}_{2.5}$ on birth weight. Maternal smoking and suspected drug or alcohol use both had positive interactions with $\mathrm{PM}_{2.5}$ on birth weight revealing a subdued association with increased $\mathrm{PM}_{2.5}$ exposure (Fig. 2a and b respectively). Maternal age was also modified by differences in $\mathrm{PM}_{2.5}$ exposure with younger maternal ages showing a larger reduction in birth weight with increased $\mathrm{PM}_{2.5}$ exposure (Fig. 2c).

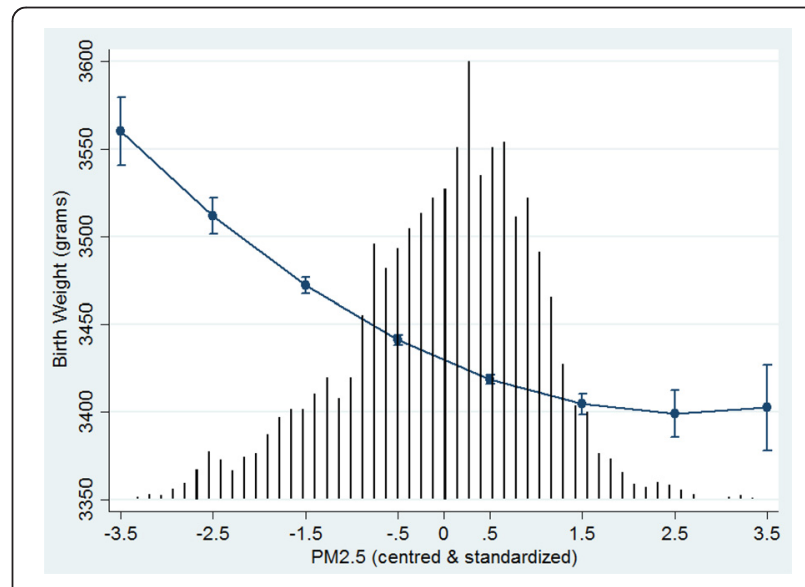

Fig. 1 Adjusted predicted effects of $\mathrm{PM}_{2.5}$ on birth weight. Predicted effects of $\mathrm{PM}_{2.5}$ on birth weight with $95 \%$ confidence intervals are conditional on model covariates included in Model 4. Black vertical lines represent the frequency distribution of $\mathrm{PM}_{2.5}$ 
Table 3 Adjusted individual and DA-level fixed effects on continuous and term birth weight and their modification by PM 2.5 (Model-4)

\begin{tabular}{llll}
\hline Variables & Main effect & Modification by PM2.5 & Corresponding figure \\
& $\beta(95 \% \mathrm{Cl})$ & $\beta(95 \% \mathrm{Cl})$ & \\
\hline PM $_{2.5}{ }^{\mathrm{a}}$ & $-22.4(-25.2--19.7)$ & $4.9(3.2-6.7)$ & 1 \\
Cigarettes/day $^{\mathrm{a}}$ & $-22.0(-23.8--20.2)$ & $2.8(2.2-3.4)$ & $2 \mathrm{~A}$ \\
Drug/Alcohol flag & $-80.6(-93.0--68.2)$ & $15.3(4.4-26.2)$ & $2 \mathrm{~B}$ \\
Maternal age & $-4.2(-6.2--2.2)$ & $5.5(3.7-7.4)$ & $2 \mathrm{C}$ \\
Gestational diabetes & $70.2(62.6-77.8)$ & $-33.8(-41.9--25.8)$ & $2 \mathrm{D}$ \\
SESi & $30.2(27.7-32.7)$ & $4.6(2.0-7.2)$ & $3 \mathrm{~A}$ \\
Immigrant density & $-33.3(-35.8--30.7)$ & $6.3(3.3-9.2)$ & $3 \mathrm{~B}$ \\
Rural address & $-29.1(-39.1--19.1)$ & $-16.4(-24.5--8.3)$ & $3 \mathrm{C}$ \\
\hline
\end{tabular}

${ }^{a}$ Modeled as a quadratic, Cigarettes/day quadratic term: 0.7(0.6 - 0.8); Model adjusted for gestational age, nulliparous, diabetes mellitus, gestational hypertension, prenatal care visits, season of birth, DA-level education

Alternatively, gestational diabetes was associated with a much greater reduction in birth weight with increasing $\mathrm{PM}_{2.5}$ compared to normal births, essentially nullifying the higher birth weights produced by the condition (Fig. 2d). Three DA-level variables showed significant effect modification with $\mathrm{PM}_{2.5}$ on birth weight. First, the interaction between SESi and $\mathrm{PM}_{2.5}$ revealed a more pronounced effect of $\mathrm{PM}_{2.5}$ in lower SES neighbourhoods (Fig. 3a). Higher Asian immigrant density buffered the $\mathrm{PM}_{2.5}$ effect (Fig. 3b), while rural DAs showed an additional reduction in birth weight with increasing $\mathrm{PM}_{2.5}$ levels compared to urban DAs (Fig. 3c).

The random effects, the explained proportional change in variance (PVC), and model diagnostics are presented in Table 5. The unadjusted ICC for the Null random-intercept model was 0.019 , indicating that $1.9 \%$ of the total residual differences in birth weight are attributable to DA-level contextual factors. The inclusion of the level-1 covariates along with the random slope for gestational age, the $\mathrm{ICC}_{\mathrm{adj}}$ (now conditional on mean-centred gestational age, 38.8 weeks),

Table 4 Sensitivity analysis using only term birth and excluding stillbirths and congenital anomalies $(N=207,405)$

\begin{tabular}{lll}
\hline Variables & Main effect & $\begin{array}{l}\text { Modification by PM2.5 } \\
\end{array}$ \\
\hline PM $95 \% \mathrm{Cl})$ & $\beta(95 \% \mathrm{Cl})$ \\
\hline Cigarettes/day ${ }^{\mathrm{a}}$ & $-24.1(-26.9--21.2)$ & $5.0(3.2-6.8)$ \\
Drug/Alcohol flag & $-21.9(-23.8--20.0)$ & $2.8(2.2-3.4)$ \\
Maternal age & $-80.1(-93.6--66.6)$ & $13.2(1.2-25.2)$ \\
Gestational diabetes & $-3.1(-5.2--1.1)$ & $5.0(3.1-6.9)$ \\
SESi & $60.2(52.1-68.4)$ & $-30.7(-39.2--22.1)$ \\
Immigrant density & $-30.5(27.9-33.1)$ & $4.3(1.5-7.0)$ \\
Rural address & $-31.2(-41.6--20.8)$ & $-16.9(-25.3--8.5)$ \\
\hline
\end{tabular}

${ }^{a}$ Modeled as a quadratic, Cigarettes/day quadratic term: 0.7(0.6-0.8); Model adjusted for gestational age, nulliparous, diabetes mellitus, gestational hypertension, prenatal care visits, season of birth, DA-level education increased to $2.3 \%$. This was due to the large reduction in the level-1 residual variance (560.5 to 435.2$)$ relative to the reduction in the level-2 random intercept variance (78.8 to 67.0). The addition of DA-level variables in Model 2 and Model 3 removed a lot of the DA-level variance reducing the $\mathrm{ICC}_{\text {adj }}$ to 1.1 and $0.8 \%$ respectively.

The level-2 random intercept standard deviation indicates that the mean birth weight for every DA has a degree of variability from the overall (BC-wide) mean birth weight. For the Null model, the overall birth weight intercept is was $3434.2 \mathrm{~g}$ with a standard deviation of 78.8. This equals a $8.6 \%$ difference in range between $95 \%$ of the DAs $(3434.2 \pm(1.96 \times 78.8)=3280.0$ and 3588.8 g). Similarly, we calculate the between-DA $95 \%$ distributional range of slopes for gestational age to fall between 255.8 and $361.6 \mathrm{~g}(308.7 \pm(1.96 \times 26.6))$, a $29.3 \%$ difference in how one week gestation increases birth weight between DAs.

The level-1 and level-2 explained PCV (L1-PCV \& L2$\mathrm{PCV}$ ) summarizes the relative degree of explained variance at the different levels between the different models. Using the Null model as the reference, the L1-model resulted in an L1-PCV \& L2-PCV of 39.7 and $27.7 \%$ respectively (Table 5 ). These are fairly large PCVs, indicating the within and between-DA variance in birth weight shown in the Null model was moderately attributable to these individual (level-1) compositional factors, largely gestational age. The addition of the DA-level variables in Model 2 explained an additional $38.3 \%$ of the DA-level variance (cumulative $\mathrm{L} 2-\mathrm{PVC}=66 \%$ ). The additions of $\mathrm{PM}_{2.5}$ and season of birth in Model 3 further explained an additional $8.5 \%$ of the level-2 intercept variance beyond that of Model 2. Model 4 accounted for an additional $2.3 \%$ L2-PCV.

Spatial analyses were used as a model diagnostic to test for significant spatial autocorrelation of the model residuals. The local Moran's I statistics reported in Table 5 




indicate the degree of local spatial autocorrelation (i.e., spatial clustering) of the level-1 (L1) residuals as well as the level-2 predicted random intercepts (L2ri) and slopes (L2rs) for all five models. Interpreted in a similar manner as a regular correlation coefficient, the Moran's I statistic reveals the existence of significant localized clustering of residuals at both level-1 and level-2 in the Null and level-1 model. The addition of level-2 variables reduced the Moran's I substantially; however small but significant clustering remained.

Sensitivity analyses using only the non-imputed DAs $\left(\mathrm{N}_{1}=228,765\right.$ in $\left.6230 \mathrm{DAs}\right)$ showed very minor differences in magnitude of significant variables in the birth weight models. In a second sensitivity analysis, we restricted the sample to only term births excluding stillbirths and congenital anomalies. As expected, there was a large reduction in the random-slope variance for gestational age due to dropping preterm births but no other differences in the observed relationships (Table 4).
Finally, a check for potential collider bias was performed by omitting gestational age as a covariate from the models [45]. Random-intercept models equivalent to those presented in Tables 2, 3 and 4 were run and assessed for differences. Many of the individual-level covariates returned to resemble their unadjusted estimates listed in Table 1. The interaction between $\mathrm{PM}_{2.5}$ and cigarettes/day remained unchanged, whereas the $\mathrm{PM}_{2.5}$ interaction with drug or alcohol flag was no longer significant $(p=0.066)$. The interaction between gestational diabetes and $\mathrm{PM}_{2.5}$ was reduced by half, but was still significant. The effects of the DA-level variables SESi and immigrant density increased moderately beyond their $95 \%$ CIs listed for Model 4. The effect for $\mathrm{PM}_{2.5}$ decreased but was still significant (-18.0 (95 \% CI -21.3 - -14.7)). The DA-level interaction between $\mathrm{PM}_{2.5}$ and immigrant density was reduced by half and marginally not significant $(p=0.051)$, while the interaction between $\mathrm{SESi}$ and $\mathrm{PM}_{2.5}$ was also reduced to non-significance ( $p=0.33$ ). 


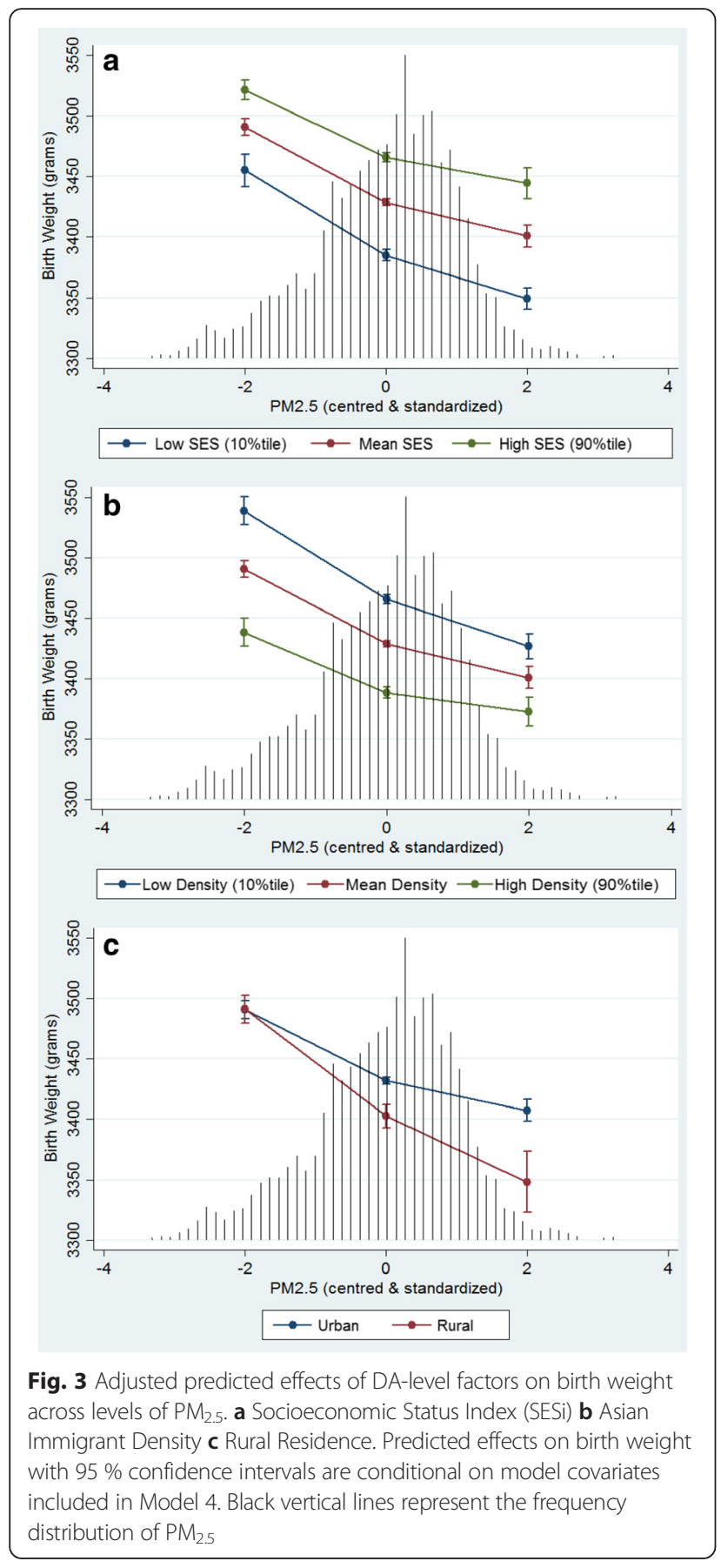

\section{Discussion}

This study employed multilevel random coefficient models to assess the effect of $\mathrm{PM}_{2.5}$ on birth weight and test its interaction with individual and neighbourhoodlevel risk factors. Our results show that individual and neighbourhood-level factors are capable of modifying the association between $\mathrm{PM}_{2.5}$ exposure and fetal growth. Furthermore, through the use of random-slopes models we show that the effect of gestational age on birth weight can vary considerably between neighbourhood DAs which was only moderately addressed in our models. After adjusting for individual-level covariates and DA-level socio-economic and socio-demographic variables, we found a significant non-linear effect between $\mathrm{PM}_{2.5}$ and birth weight in 231,929 births in British Columbia, Canada. This association was robust to the exclusion of stillbirths and congenital anomalies as well as the use of only term births and models dropping gestational age as a covariate, demonstrating that selection bias does not affect the observed $\mathrm{PM}_{2.5}$ main effects.

Our results corroborate the growing literature supporting a negative association between $\mathrm{PM}_{2.5}$ and birth weight $[3,46,47]$. Even in settings of relatively low air pollution exposure similar to our study, significant reductions in birth weight have been observed [48]. This strengthens the evidence of the low-dose effects of $\mathrm{PM}_{2.5}$ and is exemplified by Fig. 1 which shows the largest potential effects on birth weight are seen at the low to mid concentrations of $\mathrm{PM}_{2.5}$, a not uncommon dose-response phenomenon also observed in other exposure-disease contexts [49]. Other studies testing for non-linear effects of traffic-related air pollutants on fetal growth have been mixed $[23,50,51]$. Interestingly, we found a similar non-linear dose-response between cigarettes/day and birth weight, an effect also shown by England et al. using both self-reported cigarettes/day as well as urine-cotinine levels to assess exposure [52].

Our results show a negative interaction between $\mathrm{PM}_{2.5}$ and SES such that a more pronounced effect of $\mathrm{PM}_{2.5}$ was seen in lower SES neighbourhoods (Fig. 3a); however this result could be sensitive to collider bias. We also observed significant interactions between $\mathrm{PM}_{2.5}$ and Asian immigration density as well as with $\mathrm{PM}_{2.5}$ and living in a rural location (Fig. $3 \mathrm{~b}$ and $\mathrm{c}$ respectively). This suggests that that neighbourhood characteristics can not only influence fetal growth but can also modify exposures either positively or negatively. The biological mechanisms supporting such interactions have been recently reviewed [5], and have been indirectly supported in epidemiological studies that found stronger effects of $\mathrm{PM}_{2.5}$ across race, age and SES groups $[23,25,53]$. For example, the observed lower birth weights associated with neighbourhoods with higher densities of continental Asian immigrants is likely due to constitutional birth size differences [37, 38], but the positive interaction with $\mathrm{PM}_{2.5}$ may reflect the buffering effect of strong community cohesiveness and beneficial cultural practices $[23,32]$. A similar interaction was found by Basu et al. in which births to Asian mothers exhibited smaller birth weight reductions for $\mathrm{PM}_{2.5}$ constituents compared to Caucasian births [23]. Currie et al. however did not find any significant interactions between traffic-related carbon monoxide exposure and risk factors such as race, education, or low income [54]. 
Table 5 Random effects and model diagnostics from hierarchical linear models for continuous birth weight in BC, Canada

\begin{tabular}{|c|c|c|c|c|c|c|}
\hline Random effects \& model diagnostics & Null model & Null + r.slope & Model-1 (level-1) & Model-2 (SES) & Model-3 $\left(\mathrm{PM}_{2.5}\right)$ & Model-4 ( $\mathrm{PM}_{2.5}$ interact) \\
\hline \multicolumn{7}{|l|}{ Variance components } \\
\hline L1 residual (sd) & 560.5 & 442.9 & 435.2 & 435.3 & 435.3 & 435.1 \\
\hline L2 intercept (sd) & 78.8 & 67.6 & 67.0 & 45.9 & 39.7 & 37.9 \\
\hline L2 slope (sd) & - & 30.1 & 26.6 & 27.0 & 27.2 & 27.4 \\
\hline Intercept & 3434.2 & 3448.4 & 3524.2 & 3521.3 & 3523.4 & 3522.6 \\
\hline AIC & 597007 & 489148 & 480867 & 479403 & 478997 & 478753 \\
\hline L1-PCV (\%) & Ref & 37.5 & 39.7 & 39.7 & 39.7 & 39.7 \\
\hline L2-PCV (\%) & Ref & 26.4 & 27.7 & 66.0 & 74.5 & 76.8 \\
\hline$I_{C C N P C}{ }^{a}$ & 0.019 & $0.023^{a}$ & $0.023^{\mathrm{a}}$ & $0.011^{a}$ & $0.008^{\mathrm{a}}$ & $0.008^{\mathrm{a}}$ \\
\hline L1 Moran's $1^{b}$ & 0.122 & 0.108 & 0.105 & 0.043 & 0.022 & 0.018 \\
\hline L2ri Moran's I $^{\mathrm{b}}$ & 0.300 & 0.301 & 0.310 & 0.113 & 0.079 & 0.071 \\
\hline L2rs Moran's I ${ }^{b}$ & - & 0.018 & 0.018 & 0.018 & 0.017 & 0.017 \\
\hline
\end{tabular}

L1: level-1 = individual-level; L2: level-2 = DA-level; sd: standard deviation; AIC: Akaike Information Criterion; PCV: proportional change in variance; ${ }^{~ I C C: ~ I n t r a-c l a s s ~}$ correlation - is called the VPC (variance partition coefficient) when conditional on the random-slope variable, thus values in table represent intercepts for individuals with mean gestational age ( 39 weeks); L2ri: level-2 random intercept; L2rs: level-2 random slope; ${ }^{b}$ all results were significant $p<0.05$ with 999 permutations using a queen criterion spatial weight matrix

The significant negative interaction between rural address and $\mathrm{PM}_{2.5}$ may reflect the underestimation of $\mathrm{PM}_{2.5}$ in rural areas by the LUR model [34]. The composition of $\mathrm{PM}_{2.5}$, and thus its relative toxicity, is shown to vary spatially depending on its source (e.g., wood smoke vs. traffic-related emissions) and may partially explain the observed ruralurban differences $[4,6]$. The significant negative association between season of birth and birth weight could also reflect the increased presence of wood heating and vehicle exhaust in combination with winter stagnation events, but could also reflect a change in diet or increased infection rates $[4,55]$. An interaction between season of birth and $\mathrm{PM}_{2.5}$ was not statistically significant.

Interactions between $\mathrm{PM}_{2.5}$ and maternal-level variables shown to reduce birth weight independently revealed some counter-intuitive results. This included the $\mathrm{PM}_{2.5}$ interaction with maternal smoking (cigarettes/day) and with suspected drug/alcohol use where increasing $\mathrm{PM}_{2.5}$ levels tempered the negative effect of these risk factors (Fig. 2a and b). This finding was counterintuitive to our original hypothesis and published literature [54], and gave rise to the suspicion of survival bias due to competing risks (i.e., risk behaviours leading to early miscarriage, preterm or stillbirths). Although we were not able to control for fetal loss prior to 20 weeks gestation, survival bias was mitigated by using a near full population sample that included stillbirths, congenital anomalies and preterm births. Furthermore, the positive interaction between maternal smoking and $\mathrm{PM}_{2.5}$ was unchanged after the sensitivity analyses; however, the interaction between drug and alcohol use and $\mathrm{PM}_{2.5}$ remained positive but was no longer significant $(p=0.054)$. The persistence of this finding leads to a hypothesis that some individual-level exposures may act as a pre-conditioning stress that activates an adaptive response of increased biological resistance to similar stressors [56].

A protective effect of older maternal age against $\mathrm{PM}_{2.5}$ exposure was also observed by Basu et al. [23], and may stem from increased nutritional awareness among older women and/or more secure income and support networks thereby reducing potential stress and anxiety [57, 58]. Currie et al. also found significant interactions between traffic-related carbon monoxide exposure and maternal age, but that both younger (< age 19) and older (> age 34) maternal age had greater reductions in birth weight [54]. Gestational diabetes has been shown to be associated with $\mathrm{PM}_{2.5}$ and other air pollutants [59, 60]; however, their interaction with respect to birth weight has not been assessed. Our study showed that pregnancies affected by gestational diabetes had significantly higher birth weights as expected but revealed a sharp reduction in birth weight with increasing $\mathrm{PM}_{2.5}$. This significant negative interaction between $\mathrm{PM}_{2.5}$ and gestational diabetes could be related to excess of systemic or placental oxidative stress and inflammation resulting in restricted fetal growth $[15,61]$.

While the application of multilevel models in perinatal epidemiology has become more common [62], most have been random-intercept models with very few including a random-slope parameter. Permitting the slope for individual level-1 gestational age to be random can elucidate how its effect on birth weight differs between DAs. For example, the addition of level-1 covariates reduced the random-slope variability from 30.1 to 26.6. This suggests that that these maternal risk factors act through gestational age to influence birth weight and are not distributed homogeneously across DAs. In light of these findings, significant inter-DA variance remained 
for both the random intercept and slope. In other words, despite explaining a substantial proportion of the betweenDA variance in birth weight with both level-1 compositional and level-2 contextual factors, there remained unmeasured DA-level mechanisms acting either directly on fetal growth and/or through gestational age to produce between neighbourhood differences in birth weight.

Spatial analyses were used to examine the wider spatial context within which the DAs are situated and also served as a measure of model specification. The inclusion of the DA-level variables and interactions substantially reduced the spatial autocorrelation in the level-1 and level-2 random-intercept residuals (L1 \& L2ri Moran's I in Table 5). There was very little spatial autocorrelation in the level-2 random-slope parameters (L2rs Moran's I in Table 5), but it was also reduced in the DA-level models. This suggests that the census DAs perform well in capturing neighbourhood-level processes and that the selected DA-level variables do well to addess the underlying spatial processes acting on birth weight at this neighbourhood-level.

A key component of this research was the use of a landuse regression (LUR) model of air pollution [34]. While the LUR model was independently validated and achieved decent overall results in its predicted estimates, the very nature of our study design ensured some degree of exposure misclassification to our study population. Our analysis was based on maternal place of residence at delivery, and therefore intra-urban commuting and potential inter-urban relocation within the pregnancy period was not accounted for which could affect the results. Time-activity patterns show that pregnant women spend more time at home in the later stages of pregnancy, but mobility patterns may differ by age, parity and SES [63, 64]. Another limitation regarding the $\mathrm{PM}_{2.5}$ exposure assessment is that the LUR model is cross-sectional based on 2006 air quality monitoring data, while the study period of our perinatal dataset spans 6 years (2001 to 2006). We therefore assume all pregnancies were exposed to the same levels of $\mathrm{PM}_{2.5}$ for their entire pregnancy, regardless of their year of birth, based on their residential DA. While this method prevents the assessment of exposure windows by trimester, spatiotemporal studies of $\mathrm{PM}_{2.5}$ have shown little to no difference between trimester-specific and entire pregnancy effects on birth weight $[3,46,48]$. Finally, the mean $\mathrm{PM}_{2.5}$ concentrations may be underestimated by the LUR model with less variability and missing several high $\mathrm{PM}_{2.5}$ outlier locations in BC compared to compiled monitored data [26]. This could potentially result in an underestimation of our observed association of reduced birth weight with increasing $\mathrm{PM}_{2.5}$ levels.

We were unable to control for maternal-level SES, and therefore the neighbourhood-level effect estimates and interactions could reflect individual-level differences. For example, the protective effect of older maternal age buffering the $\mathrm{PM}_{2.5}$ effect on birth weight could be due to individual-level SES factors not accounted for in our models such as diet, income or stress. However, studies have found that adjustment for individual-level measures of SES did not significantly change the area-level associations $[20,65]$. Maternal education is a variable provided in the BC Perinatal Data Registry, but was only available for $10 \%$ of our population. However, the adjustment for socially-patterned behavioural risk factors such as maternal smoking, suspected drug or alcohol use and low number of prenatal care visits will control for some individual-level SES differences [66].

\section{Conclusions}

This study supports the growing literature of an effect of $\mathrm{PM}_{2.5}$ on birth weight and its modification by both maternal and neighbourhood-level factors. Most notably, it shows that lower SES neighbourhoods may be more negatively affected by higher levels of $\mathrm{PM}_{2.5}$. We observed both positive and negative interactions between maternal factors and $\mathrm{PM}_{2.5}$ that require further scrutiny but may reflect a $\mathrm{PM}_{2.5}$-oxidiative stress pathway expressed via either protective pre-conditioning or harmful overload. Targeted municipal-level interventions to reduce $\mathrm{PM}_{2.5}$ and improved neighbourhood SES may help improve birth outcomes at the population-level.

\section{Abbreviations}

BC: British Columbia; CSD: census subdivision; DA: dissemination area; ICC: intra-class correlation; L1: level-1; L2: level-2; LHA: local health area; LUR: land use regression; $\mathrm{NO}_{2}$ : nitrogen dioxide; PCV: proportional change in variance; $\mathrm{PM}_{2.5}$ : particulate matter less than 2.5 microns in diameter; PSBC: Perinatal Services British Columbia; RI: random intercept; RS: random slope; SES: socio-economic status; SESi: socio-economic status index.

\section{Competing interests}

The authors declare they have no competing interests.

\section{Authors' contributions}

AE was primarily responsible for the study design, acquisition of data, analysis and interpretation of results, drafting the article and making revisions. LA critically assessed the concept, study design, analysis and interpretation of results. LA provided critical edits of the content. Final approval of submitted manuscript was provided by both authors. LC and $\mathrm{AO}$ provided thoughtful edits on draft manuscripts and input into the manuscript framework. All authors read and approved the final manuscript.

\section{Acknowledgements}

We acknowledge Adrian Barnett in his thoughtful review and suggestions in an early draft manuscript as well as his general statistical advice. We acknowledge Eleanor Setton for her general support. We'd also like to acknowledge the staff at the Perinatal Services BC for their support regarding data access and manuscript review. All inferences, opinions, and conclusions drawn in this publication are those of the authors and do not reflect the opinions or policies of Perinatal Services BC. This publication was funded in part by the Canadian Institute of Health Research (ClHR) Operational Grant (protocol \#: 200903-202069). Funders provided no role in the design, conduct, data collection, management, analysis, interpretation, preparation, review and approval of this study.

\section{Author details}

${ }^{1}$ Division of Medical Sciences, University of Victoria, Medical Science Bld. Rm-104, PO Box 1700 STN CSC, Victoria V8W 2Y2 BC, Canada. ²Department 
of Geography, University of Victoria, David Turpin Bldg. Rm-B203, PO Box 1700 STN CSC, Victoria V8W 2 Y2 BC, Canada. ${ }^{3}$ Center for Advanced Research in Environmental Genomics, University of Ottawa, 20 Marie-Curie, Ottawa K1N 6N5 ON, Canada. ${ }^{4}$ Department of Medical Genetics, University of British Columbia, C201 - 4500 Oak Street, Vancouver V6H 3N1 BC, Canada.

Received: 24 December 2015 Accepted: 29 March 2016

Published online: 14 April 2016

\section{References}

1. Kramer MS. Determinants of low birth weight: methodological assessment and meta-analysis. Bull World Health Organ. 1987;65(5):663-737.

2. Sram RJ, Binkova B, Dejmek J, Bobak M. Ambient air pollution and pregnancy outcomes: a review of the literature. Environ Health Perspect. 2005;113:4.

3. Dadvand P, Parker J, Bell ML, Bonzini M, Brauer M, Darrow LA, et al. Maternal exposure to particulate air pollution and term birth weight: a multicountry evaluation of effect and heterogeneity. Environ Health Perspect. 2013;121(3):267-373.

4. Bell ML. Assessment of the health impacts of particulate matter characteristics. [Internet]. Boston: HEl Health Review Committee, editor. Health Effects Institute; 2012

5. Erickson AC, Arbour L. The shared pathoetiological effects of particulate air pollution and the social environment on fetal-placental development. J Environ Public Health. 2014;2014:901017.

6. HEl Review Panel on Ultrafine Particles. Understanding the Health Effects of Ambient Ultrafine Particles HEl Perspectives 3. Boston: Health Effects Institute; 2013.

7. van den Hooven EH, Pierik FH, de Kluizenaar Y, Hofman A, van Ratingen SW, Zandveld PYJ, et al. Air pollution exposure and markers of placental growth and function: the generation R study. Environ Health Perspect. 2012;120(12):1753-9.

8. Janssen BG, Munters E, Pieters N, Smeets K, Cox B, Cuypers A, et al. Placental mitochondrial DNA content and particulate air pollution during in utero life. Environ Health Perspect. 2012;120:9.

9. Veras MM, Damaceno-Rodrigues NR, Caldini EG, Maciel Ribeiro AAC, Mayhew TM, Saldiva PHN, et al. Particulate urban air pollution affects the functional morphology of mouse placenta. Biol Reprod. 2008:79(3):578-84

10. Burton GJ, Jauniaux E. Oxidative stress. Best Pract Res Clin Obstet Gynaecol. 2011;25(3):287-99.

11. Al-Gubory KH. Environmental pollutants and lifestyle factors induce oxidative stress and poor prenatal development. Reprod Biomed Online. 2014;29(1):17-31

12. Aycicek A, Ipek A. Maternal active or passive smoking causes oxidative stress in cord blood. Eur J Pediatr. 2008;167(1):81-5.

13. Kovacic $P$, Somanathan R. Mechanism of teratogenesis: electron transfer, reactive oxygen species, and antioxidants. Birth Defects Res C Embryo Today Rev. 2006;78(4):308-25.

14. Haque SK, Siddiqui MU, Islam N, Moin S. Erythrocyte markers of oxidative stress in higher age-group preeclamptic and normal pregnant mothers. Hypertens Pregnancy. 2010;29(1):69-81.

15. Kamath U, Rao G, Raghothama C, Rai L, Rao P. Erythrocyte indicators of oxidative stress in gestational diabetes. Acta Paediatr. 1998:87(6):676-9.

16. Gruenewald TL, Karlamangla AS, Hu P, Stein-Merkin S, Crandall C, Koretz B, et al. History of socioeconomic disadvantage and allostatic load in later life. Soc Sci Med. 2012;74(1):75-83.

17. Kramer MS, Seguin L, Lydon J, Goulet L. Socio-economic disparities in pregnancy outcome: why do the poor fare so poorly? Paediatr Perinat Epidemiol. 2000;14(3):194-210.

18. Blumenshine P, Egerter S, Barclay CJ, Cubbin C, Braveman PA. Socioeconomic disparities in adverse birth outcomes: a systematic review. Am J Prev Med. 2010;39(3):263-72.

19. Bird CE, Seeman T, Escarce JJ, Basurto-Dávila R, Finch BK, Dubowitz T, et al Neighbourhood socioeconomic status and biological "wear and tear" in a nationally representative sample of US adults. J Epidemiol Community Health. 2010;64(10):860-5.

20. Cubbin C, Winkleby MA. Protective and harmful effects of neighborhoodlevel deprivation on individual-level health knowledge, behavior changes, and risk of coronary heart disease. Am J Epidemiol. 2005;162(6):559-68.
21. Merlo J, Chaix B, Yang M, Lynch J, Råstam L. A brief conceptual tutorial on multilevel analysis in social epidemiology: interpreting neighbourhood differences and the effect of neighbourhood characteristics on individual health. J Epidemiol Community Health. 2005:59(12):1022-8.

22. Merlo J, Yang M, Chaix B, Lynch J, Råstam L. A brief conceptual tutorial on multilevel analysis in social epidemiology: investigating contextual phenomena in different groups of people. J Epidemiol Community Health. 2005;59(9):729-36.

23. Basu R, Harris M, Sie L, Malig B, Broadwin R, Green R. Effects of fine particulate matter and its constituents on low birth weight among full-term infants in California. Environ Res. 2014;128:42-51.

24. Généreux M, Auger N, Goneau M, Daniel M. Neighbourhood socioeconomic status, maternal education and adverse birth outcomes among mothers living near highways. J Epidemiol Community Health. 2008:62(8):695-700.

25. Zeka A, Melly SJ, Schwartz J. The effects of socioeconomic status and indices of physical environment on reduced birth weight and preterm births in Eastern Massachusetts. Environ Health Perspect. 2008;7:60.

26. Canadian Council of Ministers of the Environment. Canada-wide standards for particulate matter and ozone: five year report: 2000-2005. Winnipeg: Health Effects Institute; 2006. Report No.: PN 1374. [Accessed 19 Oct 2015]. Available from: http://www.ccme.ca/en/resources/air/pm_ozone.html.

27. Perinatal Services BC. British Columbia Perinatal Data Registry [Internet]. Vancouver; [Accessed 15 Dec 2015]. Available from: http://www. perinatalservicesbc.ca/health-professionals/data-surveillance/perinatal-dataregistry.

28. DMTI Spatial. GeoPinpoint. Ont: Markham; 2007.

29. ESRI. ArcGIS 10.2. Redlands: Environmental Systems Research Institute; 2010.

30. Gauvin L, Robitaille E, Riva M, McLaren L, Dassa C, Potvin L. Conceptualizing and operationalizing neighbourhoods: the conundrum of identifying territorial units. Can J Public Health. 2007;98 Suppl 1:S18-26.

31. Holowaty EJ, Norwood TA, Wanigaratne S, Abellan JJ, Beale L. Feasibility and utility of mapping disease risk at the neighbourhood level within a Canadian public health unit: an ecological study. Int J Health Geogr. 2010;9:21.

32. Meng G, Thompson ME, Hall GB. Pathways of neighbourhood-level socio-economic determinants of adverse birth outcomes. Int J Health Geogr. 2013;12(1):32

33. du Plessis V, Beshiri R, Bollman RD, Clemenson $H$. Definitions of "Rural". Ottawa: Statistics Canada; 2002. Report No.: 61.

34. Hystad P, Setton E, Cervantes A, Poplawski K, Deschenes S, Brauer M, et al. Creating national air pollution models for population exposure assessment in Canada. Environ Health Perspect. 2011;119(8):1123-9.

35. Jerrett $M$, Finkelstein M. Geographies of risk in studies linking chronic air pollution exposure to health outcomes. J Toxicol Environ Health A. 2005;68(13-14):1207-42

36. Chan E, Serrano J, Chen L, Stieb DM, Jerrett M, Osornio-Vargas A. Development of a Canadian socioeconomic status index for the study of health outcomes related to environmental pollution. BMC Public Health. 2015;15(1):714.

37. Shiono PH, Klebanoff MA, Graubard BI, Berendes HW, Rhoads GG. Birth weight among women of different ethnic groups. JAMA. 1986;255(1):48-52.

38. Janssen $\mathrm{P}$, Thiessen $\mathrm{P}$, Klein M, Whitfield MF, MacNab YC, Cullus-Kuhl SC. Standards for the measurement of birth weight, length and head circumfrence at term in neonates of european, Chinese and South Asian ancestry. Open Med. 2007;1(2):E74-88.

39. Graham $\mathrm{MH}$. Confronting multicollinearity in ecological multiple regression. Ecology. 2003;84(11):2809-15.

40. Canada S. Census of Canada. Topic-based Tabulations, 2006 [Internet]. Ottawa: Statistics Canada; 2006.

41. Snijders TAB, Bosker RJ. Multilevel analysis: an introduction to basic and advanced multilevel modeling. 2nd ed. London: Sage; 2012.

42. StataCorp LP. Stata 13. College Station: StataCorp LP.; 2014.

43. Anselin L. Local indicators of spatial association — LISA. Geogr Anal. 1995;27(2):93-115.

44. Skrondal A, Rabe-hesketh S. Prediction in multilevel generalized linear models. J R Stat Soc A. 2009;172(3):659-87.

45. Wilcox AJ, Weinberg CR, Basso O. On the pitfalls of adjusting for gestational age at birth. Am J Epidemiol. 2011;174(9):1062-8.

46. Stieb DM, Chen L, Eshoul M, Judek S. Ambient air pollution, birth weight and preterm birth: a systematic review and meta-analysis. Environ Res. 2012;117:100-11.

47. Pedersen M, Giorgis-Allemand L, Bernard C, Aguilera I, Andersen A-MN, Ballester $F$, et al. Ambient air pollution and low birthweight: a European cohort study (ESCAPE). Lancet Respir Med. 2013;1(9):695-704. 
48. Brauer M, Lencar C, Tamburic L, Koehoorn M, Demers P, Karr C. A cohort study of traffic-related air pollution impacts on birth outcomes. Environ Health Perspect. 2008;116(5):680-6.

49. Wigle DT, Lanphear BP. Human health risks from low-level environmental exposures: no apparent safety thresholds. PLoS Med. 2005;2(12):e350.

50. Ballester F, Estarlich M, Iñiguez C, Llop S, Ramón R, Esplugues A, et al. Air pollution exposure during pregnancy and reduced birth size: a prospective birth cohort study in Valencia, Spain. Environ Health. 2010;9:6.

51. Barnett AG, Plonka K, Seow WK, Wilson L-A, Hansen C. Increased traffic exposure and negative birth outcomes: a prospective cohort in Australia. Environ Heal BioMed Central Ltd. 2011;10(1):26

52. England L, Kendrick JS, Gargiullo PM, Zahniser C, Hannon WH. Measures of maternal tobacco exposure and infant birth weight at term. Am J Epidemiol. 2001;153(10):954-60.

53. Ebisu K, Bell ML. Airborne PM2.5 chemical components and low birth weight in the northeastern and mid-Atlantic regions of the United States. Environ Health Perspect. 2012;120(12):1746-52.

54. Currie J, Neidell M, Schmieder JF. Air pollution and infant health: lessons from New Jersey. J Health Econ. 2009;28:688-703.

55. Rasmussen SA, Jamieson DJ, Uyeki TM. Effects of influenza on pregnant women and infants. Am J Obstet Gynecol. 2012;207(3 Suppl):S3-8.

56. Calabrese EJ, Bachmann KA, Bailer AJ, Bolger PM, Borak J, Cai L, et al. Biological stress response terminology: Integrating the concepts of adaptive response and preconditioning stress within a hormetic dose-response framework. Toxicol Appl Pharmacol. 2007;222(1):122-8.

57. Newburn-Cook CV, Onyskiw JE. Is older maternal age a risk factor for preterm birth and fetal growth restriction? A systematic review. Health Care Women Int. 2005;26(9):852-75. Taylor \& Francis Group.

58. Public Health Agency of Canada. Canadian Perinatal Health Report - 2008 edition. Ottawa: Maternal and Infant Health Section; 2008. Available from: http://www.publichealth.gc.ca/cphr/.

59. Hu H, Ha S, Henderson BH, Warner TD, Roth J, Kan H, et al. Association of atmospheric particulate matter and ozone with gestational diabetes mellitus. Environ Health Perspect. 2015;123(9):853-9.

60. Malmqvist $E_{1}$ Jakobsson K, Tinnerberg H, Rignell-Hydbom A, Rylander L Gestational diabetes and preeclampsia in association with air pollution at levels below current air quality guidelines. Environ Health Perspect. 2013;121(4):488-93.

61. Qiu C, Hevner K, Enquobahrie DA, Williams MA. Maternal serum hemeoxygenase-1 (HO-1) concentrations in early pregnancy and subsequent risk of gestational diabetes mellitus. PLoS One. 2012;7(11):e48060.

62. Metcalfe A, Lail P, Ghali WA, Sauve RS. The association between neighbourhoods and adverse birth outcomes: a systematic review and meta-analysis of multi-level studies. Paediatr Perinat Epidemiol. 2011;25(3):236-45.

63. Nethery $E$, Brauer M, Janssen P. Time-activity patterns of pregnant women and changes during the course of pregnancy. J Expo Sci Environ Epidemiol. 2009;19(3):317-24

64. Bell ML, Belanger K. Review of research on residential mobility during pregnancy: consequences for assessment of prenatal environmental exposures. J Expo Sci Environ Epidemiol. 2012;22(5):429-38.

65. Luo ZC, Wilkins R, Kramer MS. Effect of neighbourhood income and maternal education on birth outcomes: a population-based study. CMAJ. 2006:174(10):1415-20.

66. Erickson AC, Arbour LT. Heavy smoking during pregnancy as a marker for other risk factors of adverse birth outcomes: a population-based study in British Columbia, Canada. BMC Public Health. 2012:12(1):102.

\section{Submit your next manuscript to BioMed Central and we will help you at every step:}

- We accept pre-submission inquiries

- Our selector tool helps you to find the most relevant journal

- We provide round the clock customer support

- Convenient online submission

- Thorough peer review

- Inclusion in PubMed and all major indexing services

- Maximum visibility for your research

Submit your manuscript at www.biomedcentral.com/submit
Biomed Central 